\title{
Archéopages
}

Archéopages

Archéologie et société

38 | 07/2013

Terres inhospitalières

\section{Optimisation par drone du levé photographique des occupations stratifiées de l'Antiquité et du Moyen Âge sur la commune de Saint-Chéron - Essonne}

\section{Vincent Goustard}

\section{OpenEdition}

\section{Journals}

Édition électronique

URL : https://journals.openedition.org/archeopages/515

DOI : 10.4000/archeopages.515

ISSN : 2269-9872

\section{Éditeur}

INRAP - Institut national de recherches archéologiques préventives

Édition imprimée

Date de publication : 1 août 2014

Pagination : 62-65

ISSN : 1622-8545

\section{Référence électronique}

Vincent Goustard, «Optimisation par drone du levé photographique des occupations stratifiées de I'Antiquité et du Moyen Âge sur la commune de Saint-Chéron - Essonne », Archéopages [En ligne], 38 07/2013, mis en ligne le 01 juillet 2015, consulté le 21 janvier 2022. URL : http://

journals.openedition.org/archeopages/515 ; DOI : https://doi.org/10.4000/archeopages.515 

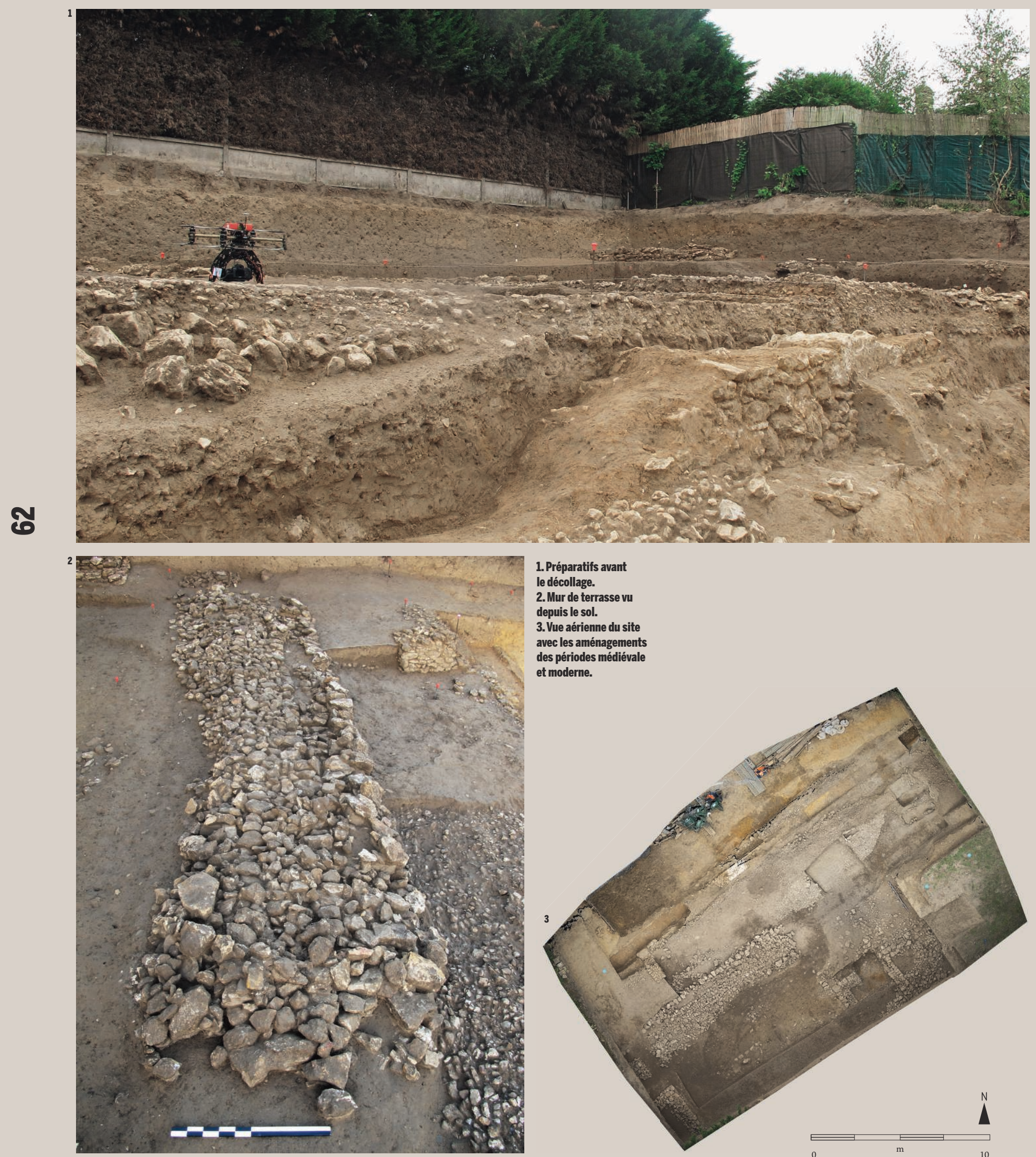

1. Préparatifs avant

le décollage.

2. Mur de terrasse vu

depuis le sol.

3. Vue aérienne du site

avec les aménagements

des périodes médiévale

et moderne.

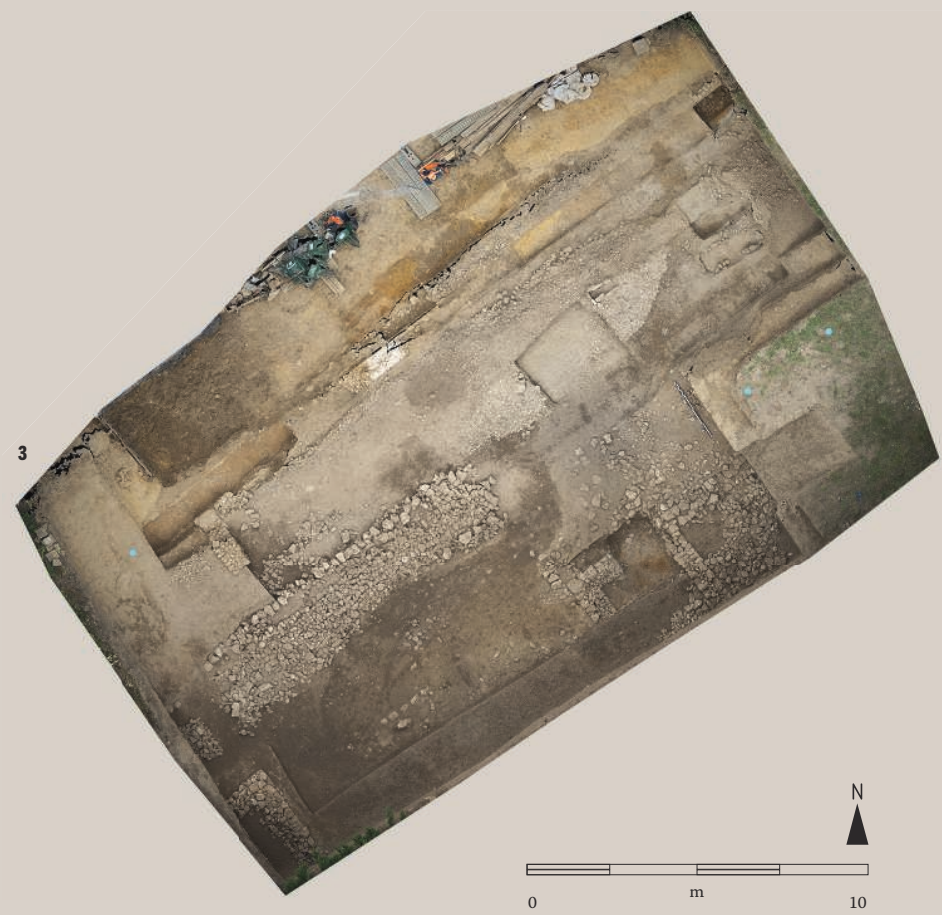


Optimisation par drone du levé photographique des occupations stratifiées de l'Antiquité et du Moyen Âge sur la commune de Saint-Chéron - Essonne

Vincent Goustard, Inrap, UMR 5060 «Institut de recherche sur les archéomatériaux»

La fouille en question concerne une petite partie d'une agglomération antique occupée entre le milieu du $\mathrm{I}^{\mathrm{er}}$ siècle et le $\mathrm{IV}^{\mathrm{e}}$ siècle de notre ère. Ponctuellement, et notamment dans le secteur de l'opération, proche du hameau de Saint-Évroult, des occupations du Moyen Âge et de la période moderne sont également représentées. La plupart des parcelles sondées ou fouillées à l'emplacement de cette agglomération, dont on estime actuellement la superficie entre 15 et 30 hectares, révèlent des occupations stratifiées. C'est notamment le cas pour la dernière intervention réalisée sur une surface de $340 \mathrm{~m}^{2}$. Le terrain en question est à l'emplacement d'une voie aménagée dans le courant du $\mathrm{I}^{\mathrm{er}}$ siècle de notre ère. Celle-ci relie les villes d'Arpajon et de Dourdan. Une autre agglomération se développe entre ces deux bourgs, à la hauteur de la commune de Saint-Chéron, sans doute en raison d'un carrefour routier avec une voie transversale. Outre la voie et ses accotements, cette fouille modeste a permis d'appréhender les façades de quelques habitations.

\section{Une première confrontation technologique}

La collaboration avec la société Captair ${ }^{\mathbf{1}}$, bien qu'expérimentale et encore balbutiante sur certains aspects, nous est apparue comme une opportunité à saisir afin de documenter l'avancement de cette fouille. Une vue générale du site ayant été provisionnée dans le budget d'opération, le recours à un drone semblait très adapté pour ce genre d'intervention. En effet, l'environnement urbain et la présence de plusieurs réseaux aériens le long du chantier n'auraient permis, dans le meilleur des cas, que des vues obliques assez déportées depuis une nacelle positionnée sur la chaussée.

Les premiers contacts ont été pris avec l'équipe au tout début des décapages du site, en juillet 2013. Deux levés photographiques y ont été effectués par la société Captair. Laéronef, équipé d'un reflex numérique à haute résolution piloté à distance, a permis de réaliser différents clichés à des hauteurs variables [ill. 1]. Cette technique peut apparaitre dans notre pratique comme une sorte d'évolution de la perche photographique développée par Patrick Neury (Inrap, région Centre). Ce dispositif a très largement concouru au renouvellement de la vision des structures archéologiques en facilitant les prises de vue zénithales. Cet outil télescopique permet par ailleurs, à partir de photos verticales et après correction des distorsions optiques, le dessin d'aménagements complexes.

Notre rencontre sur le site avec ces spécialistes a été l'occasion d'évoquer nos pratiques photographiques, nos attentes les plus immédiates et nos contraintes. Parmi ces dernières, nous avons beaucoup insisté sur la souplesse et la nécessaire disponibilité des intervenants par rapport aux variables météorologiques, la prise de vue dans de bonnes conditions étant indispensable (absence de vent fort, de pluie, présence d'une couverture nuageuse hautement souhaitable).

Calée en milieu de journée, elle nous a permis de terminer les derniers nettoyages et de bénéficier du meilleur éclairage compte tenu de la disposition du site, mal exposé, avec de fortes variations de luminosité selon les secteurs.

La première intervention sur le site a été rapide grâce à une bonne lumière et les opérations de pilotage se sont déroulées avec une extrême précision. La sécurité, qui suppose le contrôle absolu de ce type d'appareil assez lourd, a été optimale et est essentielle dans ce contexte urbanisé.

\section{La complémentarité du relevé classique et} de la vue aérienne

Ce premier levé photographique a été réalisé au terme de la fouille des aménagements médiévaux (petite zone d'inhumations, mur de terrasse, bâtiments) juste avant le démontage des maçonneries. Nous avons bénéficié très rapidement d'un premier assemblage des photos zénithales du site, qui apporte une vision complémentaire de notre lecture depuis le sol. Il nous a été fort utile pour la suite de la fouille. Ainsi, la photo imprimée a guidé l'équipe dans le démontage d'une importante structure empierrée, sorte de mur de terrasse médiéval, qui contenait les terres du versant en vue de protéger un bâtiment (chapelle ?). La vue aérienne de l'ensemble de cet aménagement a révélé une construction réalisée en plusieurs étapes alors qu'elle pouvait s'apparenter, au sol, à un simple murger.

Le seul bémol qui peut être évoqué concerne les délais d'obtention de la photo redressée du site, retard imputable semble-t-il à des problèmes de géoréférencement. Ces problèmes de calage, inhérents à la phase de test et en raison d'un matériel GPS trop sommaire, ont finalement pu être corrigés avec le concours du levé réalisé par les topographes de l'Inrap.

Malgré le panel des possibilités offertes par la photographie par drone, notamment un dessin des vestiges depuis une orthophotographie, nous avons pris le parti de conserver une approche de relevé classique compte tenu de l'aspect encore expérimental de cette prestation.

Les quelques difficultés rencontrées nous ont conforté dans nos choix. Nous avons ainsi maintenu un relevé général du site à partir de relevés sur le terrain, sur la base d'un carroyage couplé à un relevé topographique au tachéomètre.

La deuxième série de photographies a été effectuée quelques semaines plus tard lors de la fouille des aménagements gallo-romains tardifs
1. Durant l'été 2013, un

I'Inrap et la société

d'une fouille sur la

Chéron (Essonne). Olivie

Blin, AST en charge du

l'initiative de cette

expérience proposée su

plusieurs opérations

régionales à la même

période. 


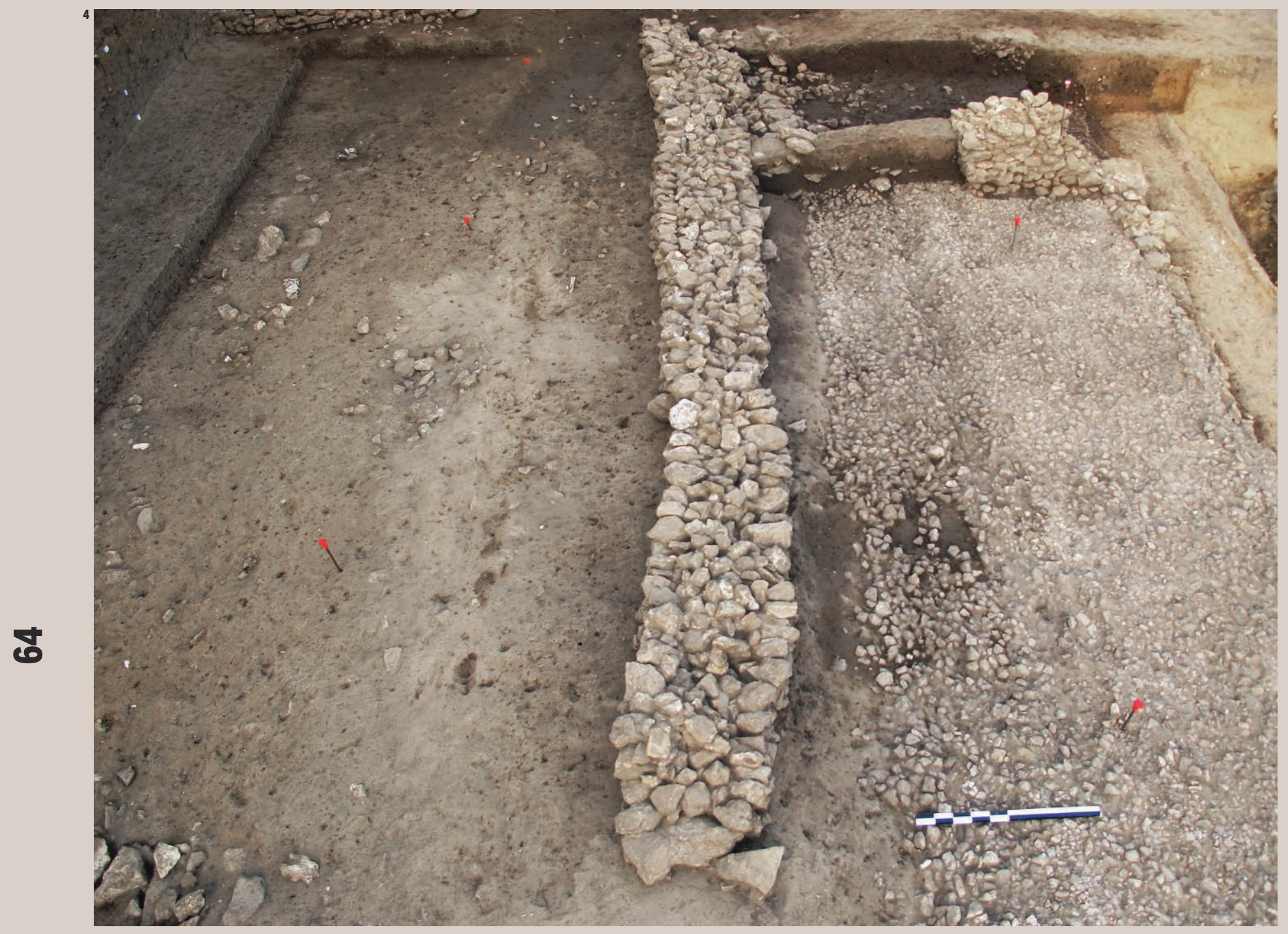

4. Démontage partiel

de l'empierrement avec

dégagement du mur originel.

Photo oblique à la perche.

5. Vue des derniers

aménagements gallo-romains.

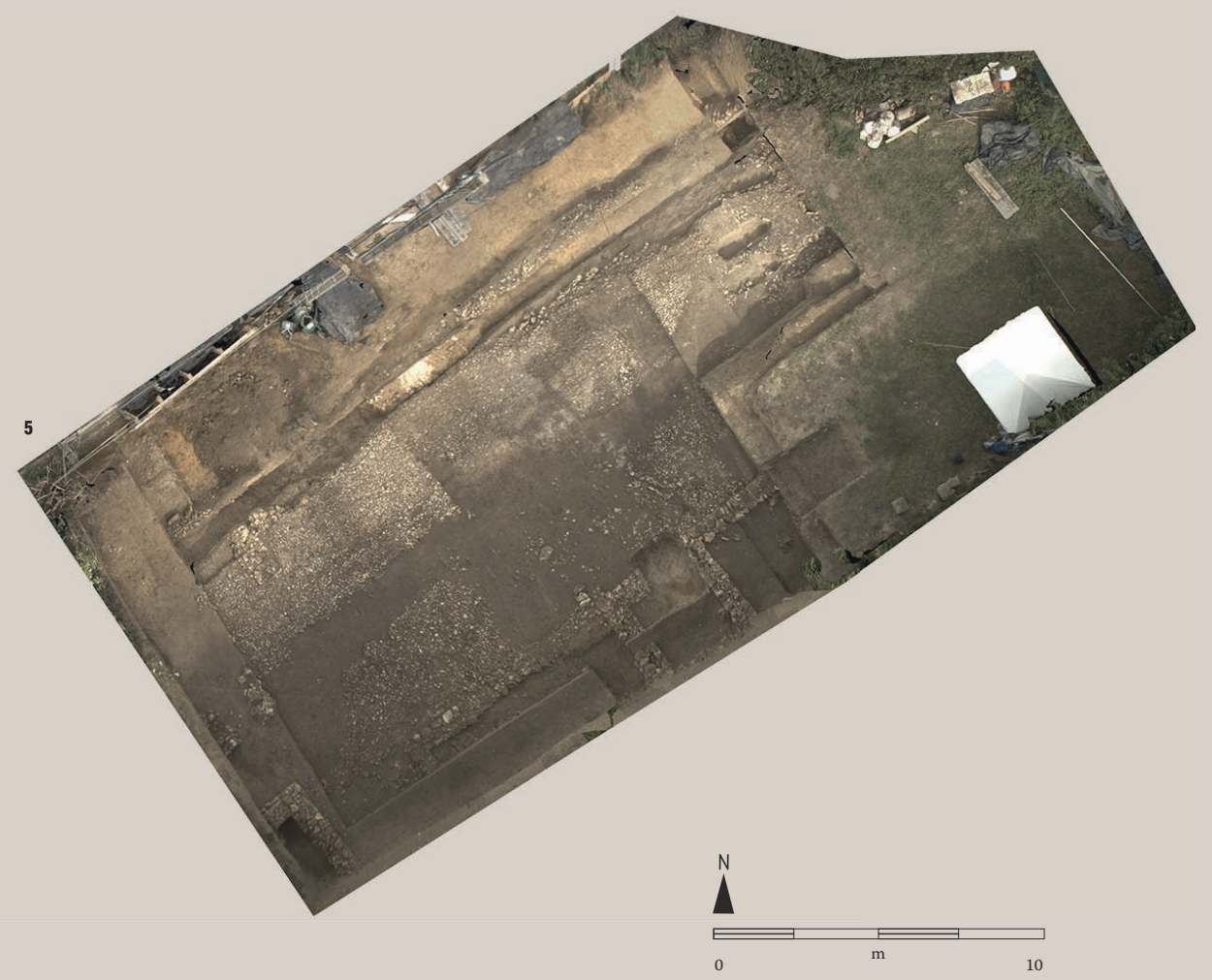


[ill. 5]. La réactivité de l'opérateur a été optimale pour ce relevé décidé en dernière minute en fonction des paramètres météorologiques et réalisé très rapidement sur le terrain avec, là encore, une grande précision. Nous avons pu récupérer en temps réel, sur le site, les photos prises, ce qui est très appréciable. On peut simplement regretter l'utilisation, pour l'assemblage de l'image corrigée, de quelques clichés réalisés au soleil, qui a généré des ombres. L'obtention de la photo redressée a été bien plus rapide que la fois précédente.

Parallèlement, la mise au point d'un serveur avec les images et des liens vers différents outils pour leur traitement sont un plus, même si nous n'avons jusqu'alors que «survolé » les applications proposées. Les études concernant cette fouille n'ont pas encore été lancées.

Le drone, un outil de communication incontestable

A posteriori, nous regrettons de ne pas avoir engagé un troisième levé à la fin de la fouille. Quelques vues obliques, ou un survol du hameau à haute altitude, quand bien même l'environnement du site et la réglementation se prêtaient difficilement à ce genre d'exercice, auraient pu aussi compléter cette couverture photographique. Nous tirons un bilan très positif de cette expérience. L'intérêt du drone comme outil de communication, dans le sens où il permet de recueillir des images originales qui illustrent et enrichissent la présentation des données de terrain, est incontestable. Ainsi, il permet de combiner des angles de vue particulièrement originaux avec une très grande souplesse d'utilisation. Le survol du chantier de Saint-Chéron, de petite taille, pouvait apparaître à de nombreux égards assez délicat par rapport à son environnement (voisinage, poteaux et réseau électrique, très hautes haies le long des emprises, route...). Le vol stationnaire et la grande stabilité de ce type d'appareil offrent très clairement de nouvelles possibilités de prise de vue dans les milieux complexes ou peu accessibles.

Il apparaît, même s'il nous reste à confronter le relevé classique des structures aux orthophotographies transmises par l'opérateur, que ces documents sont tout à fait susceptibles de renouveler et d'optimiser les approches de relevés classiques en contexte stratifié. La précision apportée est sans aucun doute très intéressante pour certains aménagements (empierrements ou autres). De même, la photo verticale haute résolution pourra peu à peu, et au cas par cas, se substituer à certains relevés. Pour autant, il nous semble que la prudence reste de mise et il convient de ne pas tomber dans les travers parfois inhérents à l'apparition de nouvelles technologies. De notre point de vue, il faut voir dans la photographie par drone un outil complémentaire et une aide dans la transmission de l'information et dans le déroulement d'une fouille par les nouveaux éclairages qu'il apporte, plus qu'une solution miracle, notamment en termes de gain de temps par rapport aux relevés manuels classiques. Ces derniers sont avant tout l'expression d'une interprétation par l'archéologue confronté à une structure, et il est sans doute dangereux et illusoire de vouloir réduire un relevé, voire un plan de site, au simple décalquage d'une photographie.

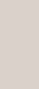

